\title{
Acquiring Syntactic Variability: The Production of Wh-Questions in Children and Adults Speaking Akan
}

\author{
Paul Okyere Omane ${ }^{* \dagger}$ and Barbara Höhle ${ }^{\dagger}$ \\ Linguistics Department, Cognitive Science Unit, University of Potsdam, Potsdam, Germany
}

OPEN ACCESS

Edited by:

Rosalind Jean Thornton,

Macquarie University, Australia

Reviewed by:

Julie Franck,

Université de Genève, Switzerland

M. Juncal Gutierrez-Mangado,

University of the Basque

Country, Spain

${ }^{*}$ Correspondence:

Paul Okyere Omane

omaneokyere@hotmail.com

tORCID ID:

Paul Okyere Omane https://orcid.org/0000-0002-0859-

9826

Barbara Höhle https://orcid.org/0000-0002-9240-

6117

Specialty section:

This article was submitted to

Language Sciences,

a section of the journal

Frontiers in Communication

Received: 10 September 2020 Accepted: 06 January 2021

Published: 26 February 2021

Citation:

Omane PO and Höhle B (2021) Acquiring Syntactic Variability: The

Production of Wh-Questions in Children and Adults Speaking Akan.

Front. Commun. 6:604951. doi: $10.3389 /$ fcomm.2021.604951
This paper investigates the predictions of the Derivational Complexity Hypothesis by studying the acquisition of wh-questions in 4- and 5-year-old Akan-speaking children in an experimental approach using an elicited production and an elicited imitation task. Akan has two types of wh-question structures (wh-in-situ and wh-ex-situ questions), which allows an investigation of children's acquisition of these two question structures and their preferences for one or the other. Our results show that adults prefer to use wh-ex-situ questions over wh-in-situ questions. The results from the children show that both age groups have the two question structures in their linguistic repertoire. However, they differ in their preferences in usage in the elicited production task: while the 5-year-olds preferred the wh-in-situ structure over the wh-ex-situ structure, the 4-year-olds showed a selective preference for the wh-in-situ structure in who-questions. These findings suggest a developmental change in wh-question preferences in Akan-learning children between 4 and 5 years of age with a so far unobserved u-shaped developmental pattern. In the elicited imitation task, all groups showed a strong tendency to maintain the structure of insitu and ex-situ questions in repeating grammatical questions. When repairing ungrammatical ex-situ questions, structural changes to grammatical in-situ questions were hardly observed but the insertion of missing morphemes while keeping the ex-situ structure. Together, our findings provide only partial support for the Derivational Complexity Hypothesis.

Keywords: Akan, wh-questions, wh-in-situ, wh-ex-situ, derivational complexity, language acquisition

\section{INTRODUCTION}

One of the essential components of a child's cognitive and social development is the acquisition of their native language(s). Acquiring a language is a complex phenomenon and research on this has become of great theoretical interest to linguists and developmental psychologists as well as for applications in diagnosis and treatment by speech therapists. This paper deals with a specific aspect of the acquisition of syntax, that is, the acquisition of wh-questions, by taking a cross-linguistic approach to children acquiring Akan-an understudied African language. So far, children's acquisition of wh-questions and yes/no questions has been investigated across many languages, such as English (Brown, 1968; Tyack and Ingram, 1977; Capdevila i Batet, 1993; Stromswold, 1995; Valian and Casey, 2003; Pozzan and Valian, 2017), French (Strik, 2012), and German (Wimmer et al., 2017). Cross-linguistic studies in this area are particularly revealing since languages show some variability in the structural properties of $w h$-questions, however this variability seems to be limited by quite strong structural conditions. Therefore, the acquisition of wh-questions provides an excellent 
window into the interplay of linguistic structure, the exposure to language-specific properties, and general cognitive mechanisms in the acquisition of a central structure in language (De Villiers, 1995). So far, research on the acquisition of wh-questions has focused on the order of learning different $w h$-words or phrases and the frequency of their usage in children of different ages see (Smith, 1933; Klima and Bellugi, 1966; Tyack and Ingram, 1977; Gazdar, 1981; Bloom et al., 1982; Hanna and Wilhelm, 1992; Stromswold, 1995; Thornton, 1995). However, the current study is devoted to the acquisition of structural aspects of question formation.

\section{Structural Variation in the Formation of Wh-Questions}

Languages of the world differ in many ways, including their phonology, morphology, and syntax. From a syntactic perspective, languages may differ in terms of headedness, placement of wh-words in wh-questions, or case assignment, among other things. For the current study, differences between languages in terms of the placement of $w h$-words or $w h$-phrases in the formation of $w h$-questions are at the center of interest. In this area, two groups of languages can be identified: 1) languages that require the $w h$-word to be moved to the left periphery of the sentence (ex-situ languages) and 2) languages in which $w h$-words remain in their basegenerated position (in-situ languages).

In some languages, the placement of the wh-word at the left periphery of the sentence is obligatory. An example of such a language is English (see 1a). Although fronting of the wh-word is the norm in English wh-questions, there are instances where the wh-word appears in non-initial position. However, this option is only possible in pragmatically highly restricted functions, for example in echo questions like (1b), which signal some surprise about or non-understanding of a previous utterance by the speaker.

\section{(1) a. What did Nancy buy? \\ b. Nancy bought what?}

In contrast, wh-words obligatorily reside in their canonical position and do not undergo any movement in so-called in-situ languages. Many languages with large numbers of speakers are classified as in-situ languages, for example Japanese, Mandarin, and Cantonese. In (2), from Mandarin Chinese, the wh-word shenme 'what' is base generated and remains in that position without any overt movement.

(2) Húfēi măi-le shénme? (Bayer and Cheng, 2015, p. 2) Hufei buy-PERF what 'What did Hufei buy?'

However, a wh-word can also appear at the beginning of a sentence in Mandarin Chinese: this can occur in cleft constructions and other topicalized sentences (see Cheung, 2008; Cheung, 2014 for details), but is not obligatory since in-situ wh-placement is possible in the same constructions.
Beyond these classic ex-situ or in-situ languages, there are also languages with greater and contextually less restricted variability in the placement of $w h$-words when forming wh-questions. Examples of such languages are French and Akan. French has both in-situ and ex-situ wh-questions with or without verb movement. This optionality offers speakers a range of alternatives in forming wh-questions (Zuckerman and Hulk 2001; Hamann, 2006; Jakubowicz, 2011; Prévost et al., 2014): a) the $w h$-word can remain in the base-generated position (see $3 a)$, b) the wh-word can be moved to the beginning of the question to the specifier position of a Complementizer Phrase (that is, Spec of CP; see 3b and 3c), c) the wh-word and the verb/ auxiliary can be moved to a pre-subject position (inversion; see $3 c$ ) or d) the wh-word can be moved to Spec CP while the auxiliary or the verb remains in the post-subject position (see $3 \mathrm{~b}$ ) without any inversion (see Zuckerman and Hulk, 2001) and references therein for a detailed analysis of French).

(3) a. Tu as fait ça comment?

You have done that how

'How did you do that?'

b. Comment tu as fait ça? How you have done that 'How did you do that?'

c. Comment as-tu fait ça? How have-you done that 'How did you do that?'

The variability in French $w h$-question formation makes the language interesting for a "thorough investigation of syntactic competence" (Hamann, 2006, p. 143) in children. The presence of this variability in the child's input allows us to test specific questions about the trajectory of children's acquisition of whquestions, that is, does this order reflect the frequency of the alternatives as children find them in their input or do structural properties independent of input frequency play a role and do children use structures that do not correspond to the adult grammar (e.g., partial movement and wh-copying)? So far, research questions like these have primarily been explored with data from French-speaking children (Hamann, 2000; Zuckerman, 2001; Zuckerman and Hulk 2001; Plunkett, 2004; Hamann, 2006; Jakubowicz, 2011, and references therein).

Most of these studies have reported that French-speaking children initially prefer to use wh-in-situ questions while whex-situ questions are largely absent from their utterances (Crisma, 1992; Hamann, 2000; Hamann, 2006, and references therein). These findings have come from both analyses of spontaneous speech (Crisma, 1992; Hulk, 1996; Hamann, 2000) and experimental studies (Zuckerman and Hulk 2001; Hamann, 2006). For instance, Zuckerman and Hulk (2001) found in an experimental study that 4- to 5-year-old children used more insitu wh-questions than adults-especially in argument compared to adjunct questions. Furthermore, children avoided the use of subject-verb inversion although this type of question was frequently produced by the adults tested in the same study. Children with developmental language disorders have been found to show a stronger preference for wh-in-situ questions 
than typically developing children across a wide age range up to 9 years of age (Hamann, 2006).

In general, in-situ wh-questions are considered to be less complex and it is assumed that their processing is less costly than the processing of ex-situ wh-questions. Children's initial preference for the in-situ structure has been linked to their less developed working memory capacity (Prévost et al., 2014). Accounts attributing a role to linguistic theory have suggested that the complexity of a question structure is especially related to the movement operations involved. This suggestion has been elaborated in the Derivational Complexity Hypothesis proposed by Jakubowicz (2004), Jakubowicz (2005).

\section{Derivational Complexity Hypothesis}

Children's language acquisition is constrained by their limited working memory capacity (Bloom, 1990) and processing difficulties that arise as a result of computational and derivational complexities. Based on the proposal that children's language development is constrained by economy considerations (Chomsky, 1995), Jakubowicz (2004) proposed the Derivational Complexity Hypothesis (DCH). The DCH claims that during language development in both typically developing children and children with atypical language development, "less complex derivations are input convergent, that is, correctly spelled out or pronounced before more complex ones" (Jakubowicz and Strik, 2008, p. 106). In this account, complexity is defined as a measure of the Derivational Complexity Metric (DCM, see 4) based on the notion of the operations of internal and external merge as captured in the following clauses:

(4) Derivational Complexity Metric (Jakubowicz, 2005) a. Merging $\alpha_{i} n$ times gives rise to a less complex derivation than merging $\alpha_{\mathrm{i}}(n+1)$ times.

b. Internal merge of $\alpha$ gives rise to a less complex derivation than internal merge of $\alpha+\beta$.

Internal merge results in a structure in which an element is moved from one position in a sentence to a different position within the same syntactic construction (see Chomsky, 2001; Citko, 2005). On the other hand, external merge combines two different elements into one structure. This type of merge is equivalent to the notion of base generation, where elements occur in their original or canonical position.

Concerning language acquisition and the development of whquestions, the DCM makes the following claims: children are sensitive to the number of times that a wh-word must merge during a derivation in order to satisfy a computational requirement. Also, the learner is sensitive to the number of constituents that can undergo internal merge when deriving a wh-question (see Jakubowicz and Strik, 2008; Jakubowicz, 2011). This suggests that $w h$-questions that do not undergo internal merge of the wh-element or only a smaller number of internal merges will be used earlier during language acquisition than questions with a higher number of internal merges (Jakubowicz and Strik, 2008). Thus, the use of in-situ whquestions and those without verb movement should precede the use of other structures in child language. The DCH is applied to the current study because it makes specific predictions for the developmental trajectory of children learning Akan.

\section{The Akan Language and Typology of Wh-Questions}

This study contributes to the research on the development of whquestions in children with novel data from Akan, which is an understudied language as far as language acquisition is concerned. Akan is interesting to look at since it also has considerable optionality in the formation of wh-questions, as in-situ and ex-situ questions are both used without any known obvious restrictions on the contextual appropriateness of one structure or the other.

Akan is a Kwa language of the Niger-Congo group of languages. It is a tone language with both lexical and grammatical tones. The canonical word order in Akan is SVO (see 5).

(5) Papa no soma-a Abofra no Man DEF send-PST child DEF 'The man sent the child.'

In Akan wh-questions, the wh-word can occur in its basegenerated in-situ position (6a) or at the beginning of the sentence in the Spec CP position (6b).

(6) a. Maame no di-i deen Woman DEF eat-PST what 'What did the woman eat?'

b. Deen na Maame no di-ie what FOC woman DEF eat-PST 'What did the woman eat?'

In addition to movement, Akan ex-situ wh-questions have two structural properties that are different from the in-situ structure. First, there is an obligatory insertion of the clitic morpheme na (Marfo and Bodomo, 2005), which is often described as a focus marker (see Boadi, 1974; Saah, 1988; Saah, 1995; Boadi, 2005; Saah, 2010). This morpheme always occurs after the wh-word in ex-situ questions. Second, the use of a resumptive pronoun (RP) may be obligatory. The RP replaces a moved noun phrase in its base position, and it is co-indexed with its antecedent (Saah, 1995; Boadi, 2005; Saah, 2010). The RP is obligatory in ex-situ whoquestions (see example 7b). It has been argued in the literature on Akan (e.g., Saah, 1995) that the RP is also present in ex-situ what-questions (see example 8), but in this case, it is covertly realized (that is, not phonetically pronounced). There are therefore no gaps at extraction sites in Akan (Saah, 1988; Saah, 1995; Saah, 2010). The realization of the RP as covert or overt is determined by the animacy status of the antecedent. A RP is covertly realized when its antecedent is [non-animate] and it is overtly realized when the antecedent is [+ animate] (Saah, 1995; Saah, 2010). 
(7) a. Maame no bo-o Hwan

Woman DEF hit-PST who

'Whom did the woman hit?'

b. Hwan na maame no bo-0 no ${ }^{1}$

who FOC woman DEF hit-PST RP

'Whom did the woman hit?'

(8) a. Maame no noa-a deen

Woman DEF cook-PST what

'What did the woman cook?'

b. Deen na maame no noa-ae $\varnothing$

What FOC woman DEF cook-PST RP

'What did the woman cook?'

Two different analyses have been proposed for the formation of ex-situ wh-questions in Akan. The first analysis assumes that the wh-element undergoes movement from its base position to the spec CP of the matrix clause. Based on this analysis, the DCM predicts that the ex-situ structure will be more complex than the in-situ structure because the ex-situ structure involves an internal merge while the in-situ structure merely involves external merge. The second analysis is motivated by the presence of the RP in the ex-situ wh-questions. In relative clauses, the presence of a RP has been described as a result of the absence of movement (Demirdache 1991; Shlonsky, 1992; Alexopoulou, 2006). Accordingly, ex-situ wh-questions with RP in Akan, can be considered as involving no movement (Saah, 1995). Based on this second analysis the DCM does not predict differences in terms of complexity between ex-situ wh-questions structure with $\mathrm{RP}$ and in-situ wh-questions.

However, following Boadi (2005), and Lartey $(2016,2019)$ we adopt the theoretical assumption that the wh-word undergoes movement from the base-generated position to the left periphery of the sentence in Akan ex-situ wh-questions. This is motivated by the fact that the clitic morpheme $n a$ is obligatory following the fronted wh-word in ex-situ questions (Marfo and Bodomo, 2005). Most importantly, as noted by Marfo and Bodomo (2005), this obligatory clitic morpheme also occurs in other structures that are generally assumed to involve movement, for example focus initial constructions. Following this analysis, we hypothesize Akan exsitu questions to be structurally more complex than in-situ questions in line with the first analysis presented above.

\section{The Present Study}

Based on the claims of the DCM, our assumption for whquestions in Akan is that the in-situ and ex-situ structures have different levels of complexity. The in-situ wh-structure only undergoes external merge at VP as a complement of the verb and is thus considered the least complex derivation. In contrast, the ex-situ wh-structure undergoes two mergeoperations: first, an internal merge by moving the wh-phrase to the Spec CP position, and second, an external merge by the insertion of the clitic morpheme which is obligatory whenever a

\footnotetext{
${ }^{1}$ The definite marker and the resumptive pronoun (RP) in Akan have the same form (no). The RP in Akan is extensively discussed in Saah (1995), Saah (2010), Boadi (2005), and references therein.
}

wh-word moves to Spec CP. Based on the DCH, we therefore expect that in-situ $w h$-questions will have a developmental advantage compared to ex-situ questions in Akan-learning children. However, according to the alternative analysis ex-situ wh-questions with RP in Akan are assumed to involve no movement (Saah, 1995). This analysis predicts no higher structural complexity for ex-situ compared to in-situ whquestions and therefore no developmental advantages for the latter.

We tested these predictions in two experiments. The first one was an elicited production task in which children were invited to ask questions about specific entities displayed in a picture. The second one was an elicited imitation task in which the participants' task was to repeat either grammatical or ungrammatical questions that were presented by the experimenter. The questions to be produced involved argument (what and who object questions) and adjunct questions (where) as previous research has shown differences in children's acquisition of argument and adjunct questions (Zuckerman and Hulk 2001; Lassotta et al., 2016). Further, a comparison between the performance for what- and whoquestions would be specifically revealing about the two alternative linguistic analyses since the DCM would not predict any differences between these two question types while the alternative analysis may, due to the overt presence of the RP in the who- but not in the what-questions. As mentioned above, both the in-situ and ex-situ question structures are commonly used by Akan-speaking adults with no contextual limitations, so that children probably receive regular input of both of these structures. Since adults' preference for one of the question types has not been investigated so far, Akan-speaking adults were also tested in the experiments. Therefore, the present study not only adds to our knowledge on the acquisition of optionality in whquestions, but it is also informative about the use of different question structures in adult speakers of Akan.

\section{STUDY 1: QUESTION ELICITATION TASK}

\section{Methods \\ Participants}

Forty-four Akan-speaking children and 22 adult native speakers (range: 24-48 years) of Akan participated in this study: twentyone 4-year-olds $(\mathrm{M}=4.4$ years; $\mathrm{SD}=3.30$; range: $4 ; 0-4 ; 11)$ and twenty-three 5 -year-olds $(M=5.3$ years; $S D=3.64$; range: $5 ; 0-5$; 11). All children were recruited from two different kindergarten and primary schools in Ghana. Oral reports by parents and teachers indicated that none of the children had any hearing difficulty, articulation problems, or showed any other sign(s) of atypical development. Teachers and parents gave written informed consent that their students and children could participate in the experiment. No child was tested without the full approval of either the teacher or the parent. The adult group was comprised of parents either of children who participated in the study or of children who attended the same school but were not included in the study because they fell outside the age range. In addition, teachers from the children's schools participated. The 
headmaster of each school informed parents about our study and if they agreed to take part in the study, we contacted them to arrange a meeting. About $20 \%$ of the adult participants were monolingual speakers of Akan. The others were English-Akan bilinguals and English-Akan-Ewe multilinguals. These participants typically speak Akan in their daily life, which is the dominant language of the region and the community where we conducted our study. Monolingual speakers are rarely encountered in Ghana, which is a multilingual country with over fifty spoken languages (Bodomo et al., 2009; BollAvetisyan et al., 2020).

\section{Stimuli}

Pictures were used as visual stimuli to elicit the production of questions that involved three different wh-pronouns: hwan/ hena 'who', $(\varepsilon)$ deen 'what', and $(\varepsilon)$ hen/henfa 'where'. The whatand who-questions were all object questions. Forty-five pictures with corresponding sentences (prompts; see examples 9-11) were used in this elicitation task. Following a paradigm introduced by Crain and Thornton (1998), the prompts were meant to elicit specific questions from the participants which they should address to the second experimenter present during the experiment, whose name was Kwame. Each of the three interrogative pronouns was tested with 15 pictures and the corresponding prompts.

(9) Bisa Kwame baabi a okra no ehye

Ask Kwame location that cat DEF be.locate

'Ask Kwame about the location of the cat.'

(10) Bisa Kwaame adec a papa no re-pia

Ask Kwame thing that man DEF PROG-push

'Ask Kwame about the thing that the man is pushing.'

(11) Bisa Kwaame dec maame no re-dware no

Ask Kwame the person woman DEF PROG-bath RP

'Ask Kwame about the person the woman is bathing.'

None of the verbal prompts involved a wh-word. The pictures used to elicit what-questions showed at least one animate entity (an Agent) involved in some activity with an object (e.g., a man pushing a car). For who-questions, the pictures showed two animate entities: an Agent and a Theme/ Patient engaged in some form of activity (e.g., a woman bathing a baby). For where-questions there was one animate entity at a given location and the task of the participants was to ask about the location of the animate entity (e.g., a cat hiding in a box).

\section{Procedure}

Both children and adults were tested individually, either in the schools or at home. The experiment was run in two sessions. Each session lasted for about $15 \mathrm{~min}$. There was a break of at least 5 min between the two sessions to give the children the chance to take a little rest, visit the washroom, and also to be attended to in other ways. Written informed consent was obtained before the experiment began. A language background questionnaire only for the children was also filled in by the parent/caretaker or teacher after completion of the informed consent form. The language background questionnaire was used to gain information about the language(s) spoken by the child, and potential hearing or articulation problems. The experiment began with a practice session to ensure that participants became familiarized with the procedure. All instructions for the experiment were read out loud to the participants by the researcher in the Akan language. Participants were instructed to ask questions based on the pictures that were shown on the computer screen and the verbal prompt that accompanied the visual presentation. Visual stimuli were presented on a MacBook Pro laptop using the OpenSesame program (3.2.6 Kafkaesque Koffka release) while the sentence prompts were presented verbally by the researcher. Participants sat next to the experimenter and both faced the computer screen during stimulus presentation and recording of the participants' responses. The participants' responses were audio recorded using Audacity (version 2.3.1.0) installed on the MacBook Pro laptop. An external microphone was connected to the laptop to aid the recording. We used the same procedure to test both children and adults, except that in the case of adults the experiment was carried out in only one session. The 45 experimental trials were presented in a mixed order.

\section{Coding and Scoring}

All audio recordings were transcribed by two native speakers of Akan. The coders annotated each participant's response as either an in-situ or an ex-situ wh-question. An answer was coded as an in-situ wh-question if the $w h$-word occurred in its base-generated position. An answer was coded as an ex-situ wh-question if the $w h$-word occurred at the beginning of the question and preceded the clitic morpheme na.

\section{RESULTS}

Responses that were not appropriate concerning the presented picture and prompt were excluded from further analysis. The incorrect responses were responses where participants used the wrong $w h$-word (i.e., who-questions were produced as what- and where-questions, what-questions were produced as where- and who-questions, and where-questions were produced as whatquestions), or null responses where participants did not respond at all. The excluded responses amounted to $10.4 \%$ of the total responses given. Overall, the two child groups and the adults produced a total of 2,666 (89.8\%) correct in-situ and ex-situ responses across the three pronoun conditions. Adults produced 969 correct responses: 322 (33.2\%) in-situ questions and 647 (66.8\%) ex-situ questions. The 4-year-olds produced 761 correct responses: 339 (44.5\%) in-situ and 422 (55.5\%) ex-situ questions. The 5-year-olds produced 936 correct responses: 563 (60.1\%) insitu questions and 373 (39.9\%) ex-situ questions. Both adults and children produced a considerable high number of both ex-situ and in-situ wh-questions (Figure 1).

We analyzed the data with R ( $\mathrm{R}$ Core Team, 2018) using Generalized Mixed Effects Model with the glmer function from the package lme4 (Bates et al., 2015). Our model comparisons were tested using the likelihood ratio test. The lowest Akaike 


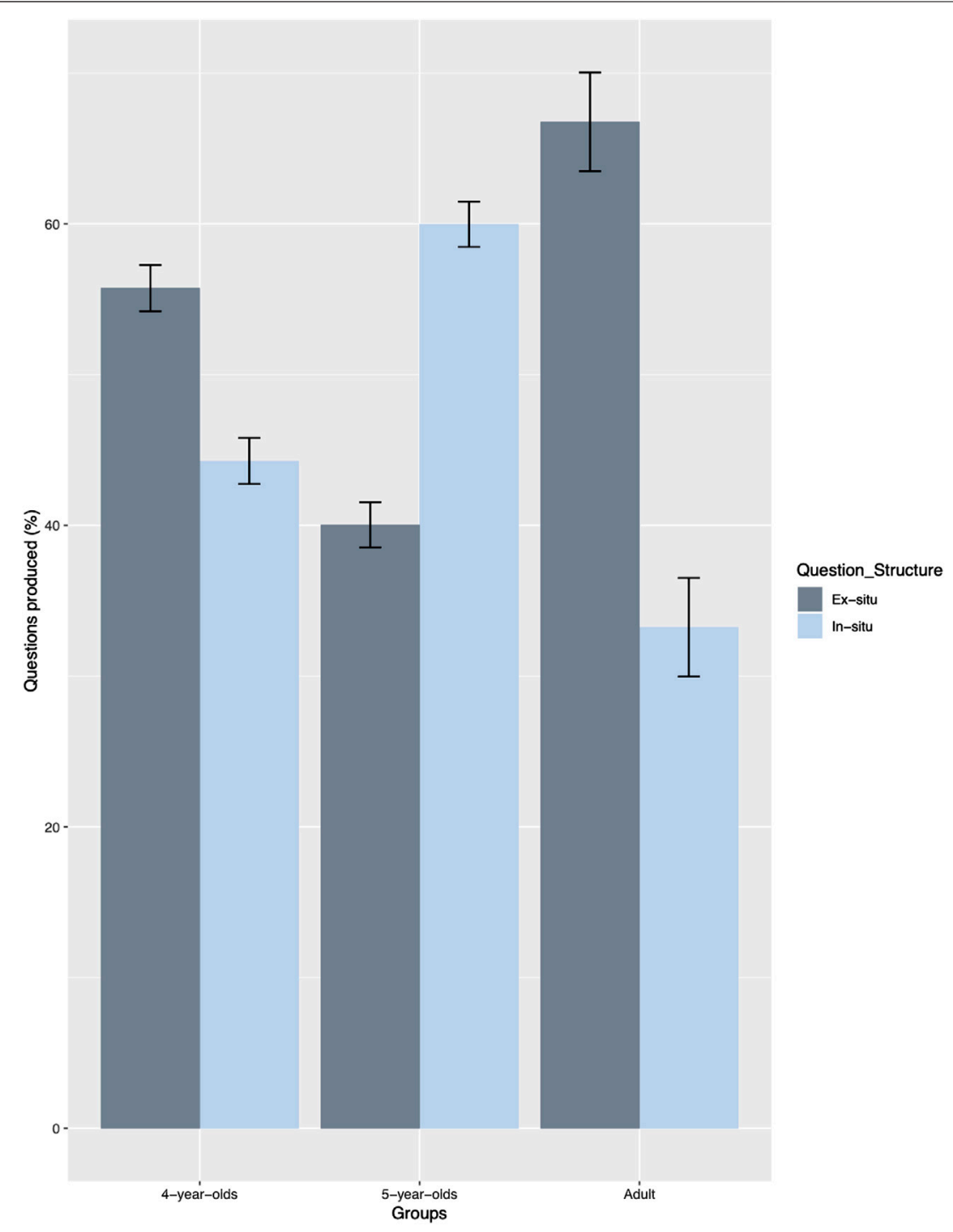

FIGURE 1 | Percentages of in-situ and ex-situ questions produced by the three different age groups. Error bars indicate a 95\% confidence interval.

Information Criterion (AIC, Akaike, 1998) and the significant difference in the Chi-square test were used to determine the best fitting model using the anova function. The model with Condition (what- and where- and who-questions) and age (4-years, 5-years and adults) as fixed factors (coded as categorical variables), and participants and items as random factors was the best fitting model. In this model, response type (in-situ structure or ex-situ structure) were entered as dependent variable where in-situ structure is coded as 0 and ex-situ structure as 1 . We used contrast coding, which sets the 4-year-olds and what-questions as the reference groups for age and condition respectively. Thus, the intercept contains the reference level for age and condition. Table 1 provides a summary of the best fitting model. Compared to the best fitting model, the model with interaction of age and condition as fixed factors and participant and item as random factors showed that there is no interaction of age and condition as the Chi-square test result is not significant $\left(\chi^{2}(4)=4.915, p=0.296\right)$. The main effect of age on participants' choice of structure shows that the 5-year-olds used significantly more in-situ structures than the 4-year-olds. However, adults' use of the in-situ structure differs only marginally from the 4-year-olds. The effect of condition on participants' choice of structure revealed a significant difference between what-questions and where-questions and between whatquestions and who-questions (Figure 2).

TABLE 1 | Summary of model output on response type in experiment 1.

\begin{tabular}{lcccc}
\hline Fixed effects & Estimate & SE & z-value & $\operatorname{Pr}(>|z|)$ \\
\hline Intercepts & 0.3081 & 0.255 & 1.209 & 0.227 \\
5-year-olds & -0.7490 & 0.338 & -2.214 & $<0.05^{\star}$ \\
Adults & 0.6230 & 0.343 & 1.815 & 0.069 \\
Where-questions & -0.2864 & 0.104 & -2.749 & $<0.01^{*}$ \\
Who-questions & 0.2490 & 0.111 & 2.251 & $<0.05^{\star}$
\end{tabular}




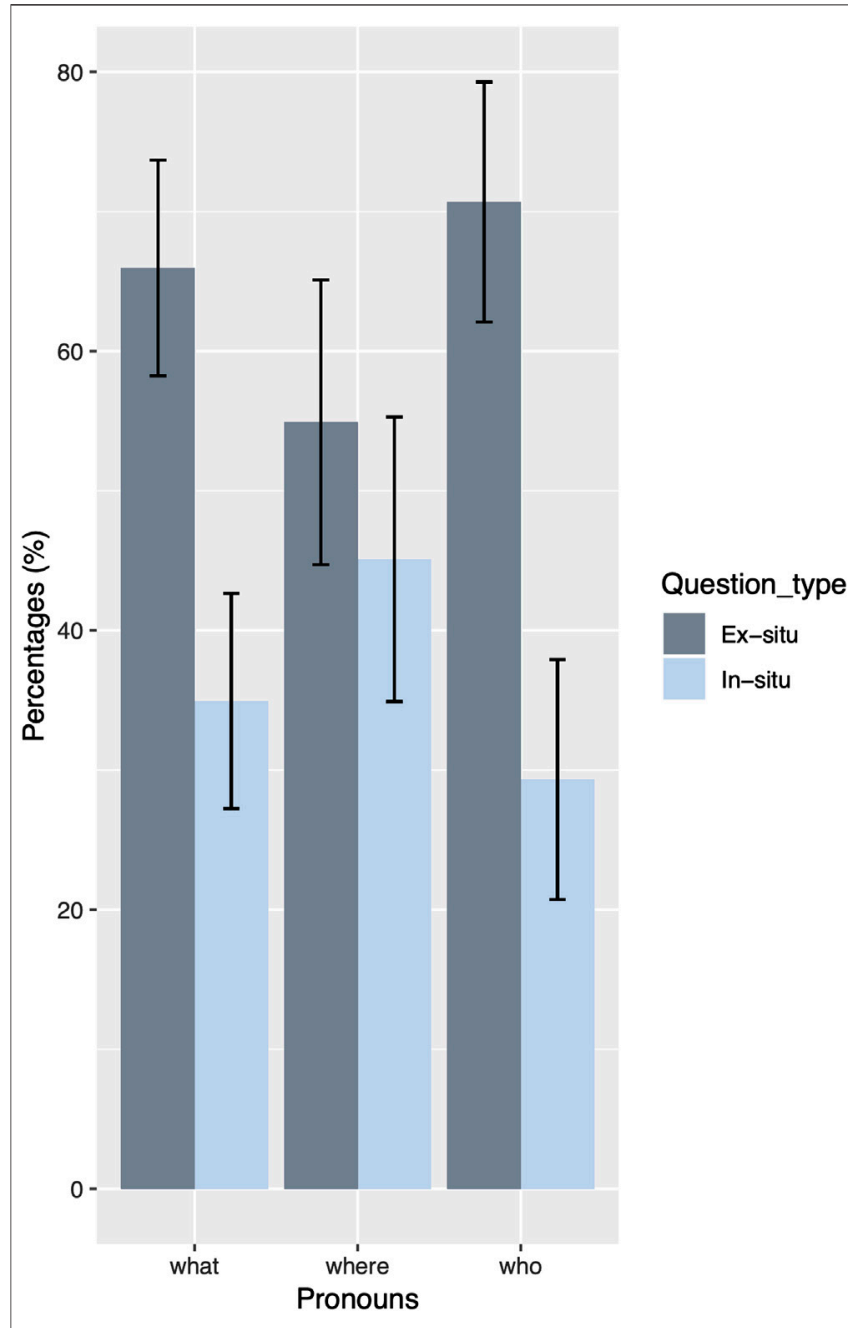

FIGURE 2 | Proportions of ex-situ and in-situ questions in the different conditions. Error bars indicate a 95\% confidence interval.

A post-hoc pairwise comparison with the emmeans package (Lenth, 2020) in R confirms that the 4-year-olds and adults do not differ significantly in their amounts of in-situ questions produced $(\beta$ $=-0.623, \mathrm{SE}=0.343$, z.score $=-1.815, p=0.165)$. However, the 4and the 5-year-olds differ marginally in their production of in-situ questions $(\beta=0.749, \mathrm{SE}=0.338$, z.score $=2.214, p=0.067)$, while the 5 -year-olds produce significantly more in-situ questions than the

TABLE 2 | Model summary on exact repetition in the grammatical and ungrammatical conditions in experiment 2.

\begin{tabular}{lcccc}
\hline Fixed effects & Estimate & SE & z-value & $\operatorname{Pr}(>|z|)$ \\
\hline Intercept & 7.4392 & 0.8545 & 8.706 & $<0.001^{\star \star \star}$ \\
5-year-olds & -1.8994 & 1.5171 & 1.252 & $<0.5$ \\
Adults & -2.4141 & 0.9974 & -2.420 & $<0.05^{\star}$ \\
Ungrammatical & -9.1072 & 0.8294 & -10.981 & $<0.001^{\text {*** }}$ \\
5-years:Ungrammatical & -0.9155 & 1.4845 & -0.617 & $=0.54$ \\
Adults:Ungrammatical & -4.1355 & 1.5333 & -2.697 & $<0.01^{\text {** }}$
\end{tabular}

adults $(\beta=-1.372, \mathrm{SE}=0.335$, z.score $=-4.096, p<0.001)$. Post-hoc pairwise comparisons with the emmeans package (Lenth, 2020) in $\mathrm{R}$ showed that participants produced fewer in-situ questions in whoquestions than in where-questions $(\beta=-0.535, \mathrm{SE}=0.111$, z.score $=$ $-4.841, p<0.001)$ and also fewer in-situ questions in what-questions than in where-questions $(\beta=0.286, \mathrm{SE}=0.104$, z.score $=2.749, p<$ $0.05)$. Only a marginal difference occurred between what- and whoquestions $(\beta=-0.249, \mathrm{SE}=0.111$, z.score $=-2.251, p=0.063)$.

\section{DISCUSSION}

The elicited production task tested the production of wh-questions in Akan-speaking children and adults. Overall, we found that the children as well as the adults used both types of structures in this task although with slight differences in their proportions. The comparison between the groups showed that the 4-year-olds did not differ from adults in their amounts of in-situ questions, but they produced marginally fewer in situ-questions than the 5-year-olds. However, 5-year-olds differed from adults in their question formation: they produced significantly more in-situ questions than adults. Overall, this pattern suggests that 4-year-olds and adults show a preference for producing ex-situ questions while the 5-year-olds show a reverse pattern-although statistically verified only in comparison to adults.

So, far the results of our study allow for several conclusions. The results from our adult group clearly suggest that adult speakers of Akan have a preference for producing ex-situ compared to in-situ questions in the elicited production task applied in our experiment. We had no clear expectation which structure would be preferred by the adults, but the results are clear-cut. Interestingly, this is in line with what other studies report for French adults and raises the question why overall there seems to be a preference for the structurally more complex in-situ variant. With respect to language acquisition, two quite remarkable findings emerge from our data. The first one is that even in the youngest age group both structures are produced, suggesting that children at this age have both structures in their linguistic repertoire. In addition, in this younger age group no evidence for a preference for the less complex in-situ structure could be found, - a result that is not in line with the predictions of the DCH (Jakubowicz, 2004; Jakubowicz, 2005) which predicts an initial stronger preference for the less complex in-situ structure. Contrary to the $\mathrm{DCH}$, the 4-year-olds showed a tendency to produce the ex-situ structure albeit only in whoand what-questions. Surprisingly, children's preferences for one question structure or the other seems to be drifting with age as the 5-year-olds showed a higher tendency along a preference for the in-situ questions. With this pattern they were different from the adults but marginally different from the younger children. Before we discuss these surprising findings more closely, we will first report the results from our second task to examine how robust this pattern is across different tasks.

The preference for in-situ vs. ex-situ questions was also modulated by the wh-word: the what- and who-questions more often were realized as in-situ structures than the where-questions while no difference occurred between the what - and the who-questions. This may suggest that a 
preference for in-situ vs. ex-situ questions in Akan may be affected by the status of a question as an argument or an adjunct question. This resembles findings from French speaking adults who judged the acceptability of in-situ and ex-situ adjunct and argument simple questions as slightly different (Lassotta et al., 2016). In this study, argument exsitu and in-situ argument questions did not differ significantly in their acceptability, but adjunct ex-situ questions were more often judged as acceptable than in-situ questions. However, the data from Akan seem to go into the other direction since the difference between ex-situ and in-situ structure seems to be smaller in the adjunct questions compared to the argument questions.

\section{STUDY 2: QUESTION REPETITION TASK}

To check whether the structure preference observed in the first study was connected to the experimental materials and procedure used or could be verified with another method, we conducted an elicited imitation task. Here, participants were presented with grammatical and ungrammatical wh-questions and asked to repeat and correct them if they considered them incorrect. Previous studies have shown that elicited imitation tasks are able to reveal young children's grammatical knowledge in two ways: first, grammatical sentences are more often repeated literally than ungrammatical sentences and second, ungrammatical sentences are often changed to their grammatical counterpart (e.g., Höhle et al., 2001). Accordingly, two questions were pursued with the current experiment: First, if both question structures are part of the participants' linguistic repertoire, grammatical questions should be repeated exactly more often than ungrammatical questions, independent of the specific structure (in-situ vs. ex-situ). Second, the ungrammatical questions used in this study could be corrected in two different ways, resulting in either the in-situ or the ex-situ structure. The choice between these alternative corrections should therefore also be informative about the preferred question structure in the children of the ages tested and in the adults.

\section{Methods \\ Participants}

All children and adults who had participated in the experiment from Study 1 also took part in this experiment. No additional participants were recruited.

\section{Stimuli}

Fifty-four questions (30 grammatical and 24 ungrammatical ones) were constructed for this study. We focused on the same three $w h$ pronouns that were used in Study 1. For each pronoun, there were ten grammatical questions: 5 with the in-situ structure and 5 with the ex-situ structure, making a total of 30 stimuli across the three pronoun conditions. In-situ grammatical questions began with a noun and ended with the wh-word under investigation (see 12), which reflects the canonical SVO order in Akan. Ex-situ grammatical sentences began with a $w h$-word and ended with a verb (for what- and where-questions (see 13a)) or a RP (for whoquestions (see 13b)).

(12) Abofra no re-di deen

child DEF PROG-eat what

'What is the child eating?'

(13) a. edeen na abofra no re-di what FOC child DEF PROG-eat

'What is the child eating?'

b. Hwan na maame no bo-o no

who FOC woman DEF beat-PST RP

'Whom did the woman beat?'

All ungrammatical questions were based on the ex-situ structure, that is, the structure with an initial wh-word. To make them ungrammatical, the clitic morpheme na, which is obligatory in Akan ex-situ questions, was omitted (see 14a-c). In the case of who-questions (see 14c), the obligatory RP was also omitted.

(14) *a. $\operatorname{chen}(\mathrm{fa}) \_$abofra no wo
Where child DEF be.locate
'Where is the child?'
^b. Deen Kofi di-i
What Kofi eat-PST
'What did Kofi eat?'
${ }^{*}$ c. Hwan Kof bo-o
Who Kofi beat
'Whom did Kofi beat?'

If corrected, the ungrammatical questions could be changed in two ways into their grammatical counterparts: either by replacing the structure with an in-situ question or by keeping the ex-situ structure and adding the clitic morpheme $n a$ and the RP in the case of a who-question.

\section{Procedure}

Participants were tested individually directly after the completion of the experiment from Study 1. This second experiment lasted for approximately $10 \mathrm{~min}$. All participants were instructed to repeat the questions and were told that they should correct them if they hear one that they think is incorrect. All grammatical and ungrammatical sentences were presented by the experimenter. The participants' responses were audiorecorded using Audacity (version 2.3.1.0) installed on a MacBook Pro laptop. The MacBook Pro laptop was connected to an external microphone to support the recording. The 54 experimental stimuli were pseudorandomized so that participants would not hear two or more questions from the same pronoun condition, or two or more grammatical or ungrammatical questions consecutively. Participants were allowed to make selfcorrections as many times as they wanted whenever they realized that they had made a mistake. However, they were never prompted by the experimenter to reconsider their response. 


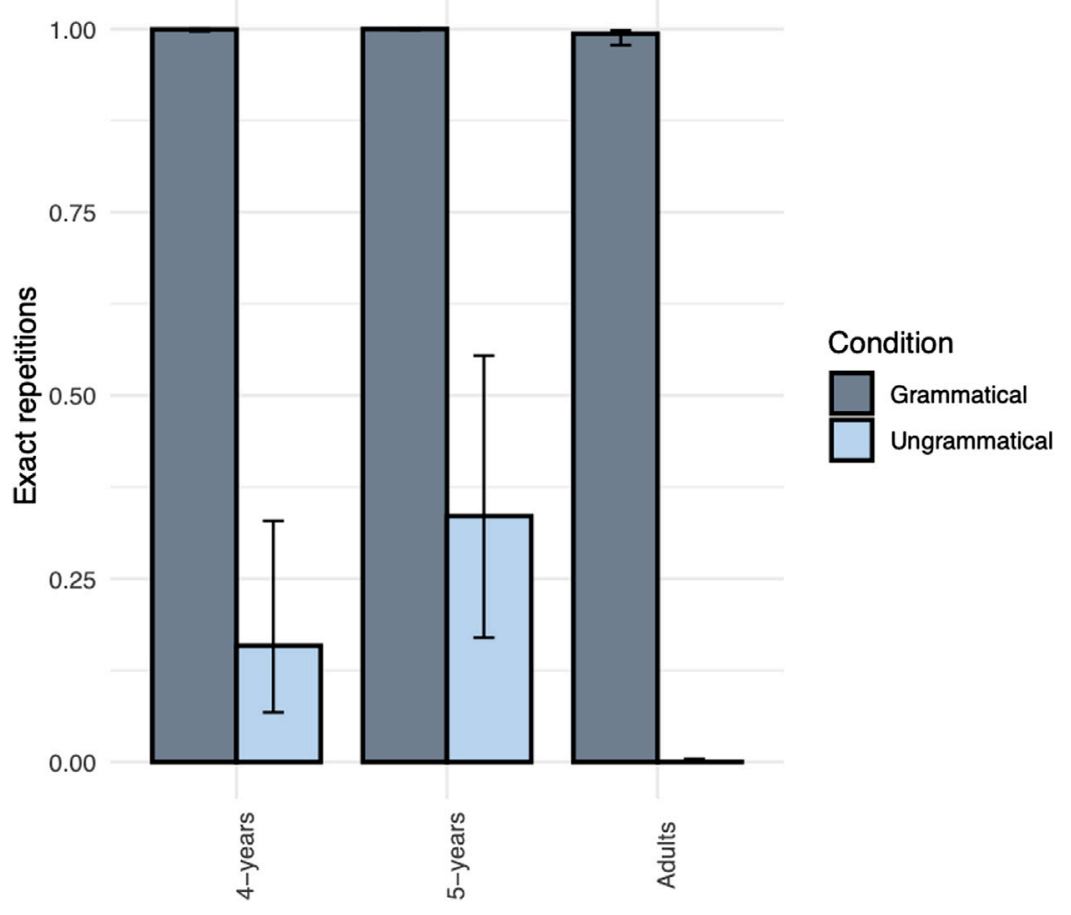

FIGURE 3 | Proportions of exact repetitions produced in the grammatical and ungrammatical conditions by the different groups. Error bars indicate a 95\% confidence interval.

\section{Coding and Scoring}

All audio recordings were transcribed by two native speakers of Akan. Since participants were allowed to correct themselves whenever they deemed necessary, we coded only their last response. For all grammatical questions, the participants' responses were annotated as exact repetition when a literal repetition was produced, while a change from the ex-situ to the in-situ structure and vice versa was coded as a switch. In case of the ungrammatical questions, an insertion of the clitic morpheme for all question types and additionally the RP for only who-questions while maintaining the ex-situ structure was coded as insertion.

\section{RESULTS}

Figure 3 shows the percentages of exact repetitions in the grammatical and ungrammatical sentences produced by the three different groups.

A Generalized Mixed Effects Model with participants' responses as dependent variable $(1=$ exact repetition $/ 0=$ non exact repetition), Age of participants (4-/5-year-olds/Adults) and Condition ( $1=$ Grammatical $/ 0=$ Ungrammatical sentences $)$ as categorical fixed factors and their interactions. 4-year-olds responses to grammatical questions were assigned to the intercept. The random factors included participants and subjects with random slopes. The summary statistics of the best-fitting model are reported in Table 2 and show a significant Intercept, a significant effect of Age (4-year-olds when compared to Adults), an effect of Condition as well as an interaction between Age (4year-olds and Adults) and Condition. To interpret the significant interactions, we ran Bonferroni adjusted pairwise comparisons with estimated group means using the 'emmeans' package in $\mathrm{R}$. When comparing each age groups' performance on the grammatical questions with that on ungrammatical sentences we found that all groups were less likely to produce exact repetitions of ungrammatical than of grammatical sentences (4-year-olds: $\beta=9.11, \mathrm{SE}=0.829$, z.score $=10.981, p<0.0001$; 5-year-olds: $\beta=10.02, \mathrm{SE}=$ 1.452, z.score $=6.902, p<0.0001$; adults: $\beta=13.24$, $\mathrm{SE}=$ $1.453, \mathrm{z}=9.111, p<0.0001)$. The comparison between the age groups showed that the probability of participants responding with an exact repetition of the grammatical questions (rather than with a non-exact repetition) did not differ significantly by age $(p>0.05)$. However, we did find significant differences in participants' performance in the ungrammatical condition: both child groups were more likely to produce exact replications of the ungrammatical sentences than adults (4-year-olds-adults: $\beta=6.550$, SE $=1.457$, z.ratio $=4.495, p<0.0001$; 5-yearolds—adults: $\beta=7.534, \mathrm{SE}=1.466$, z.ratio $=5.138, p<0.0001$ ). However, 4- and 5-year-olds did not differ significantly in producing exact repetitions in the ungrammatical condition $(\beta=-0.984, \mathrm{SE}=0.663$, z.ratio $=-1.484, p=0.4138)$.

Figure 4 presents the proportions of the three response types observed in the ungrammatical question condition. Remember, that the ungrammatical questions could be corrected in two ways to form a grammatical question: either by the insertion of the clitic morpheme na and the RP (in case of a who-question) while 


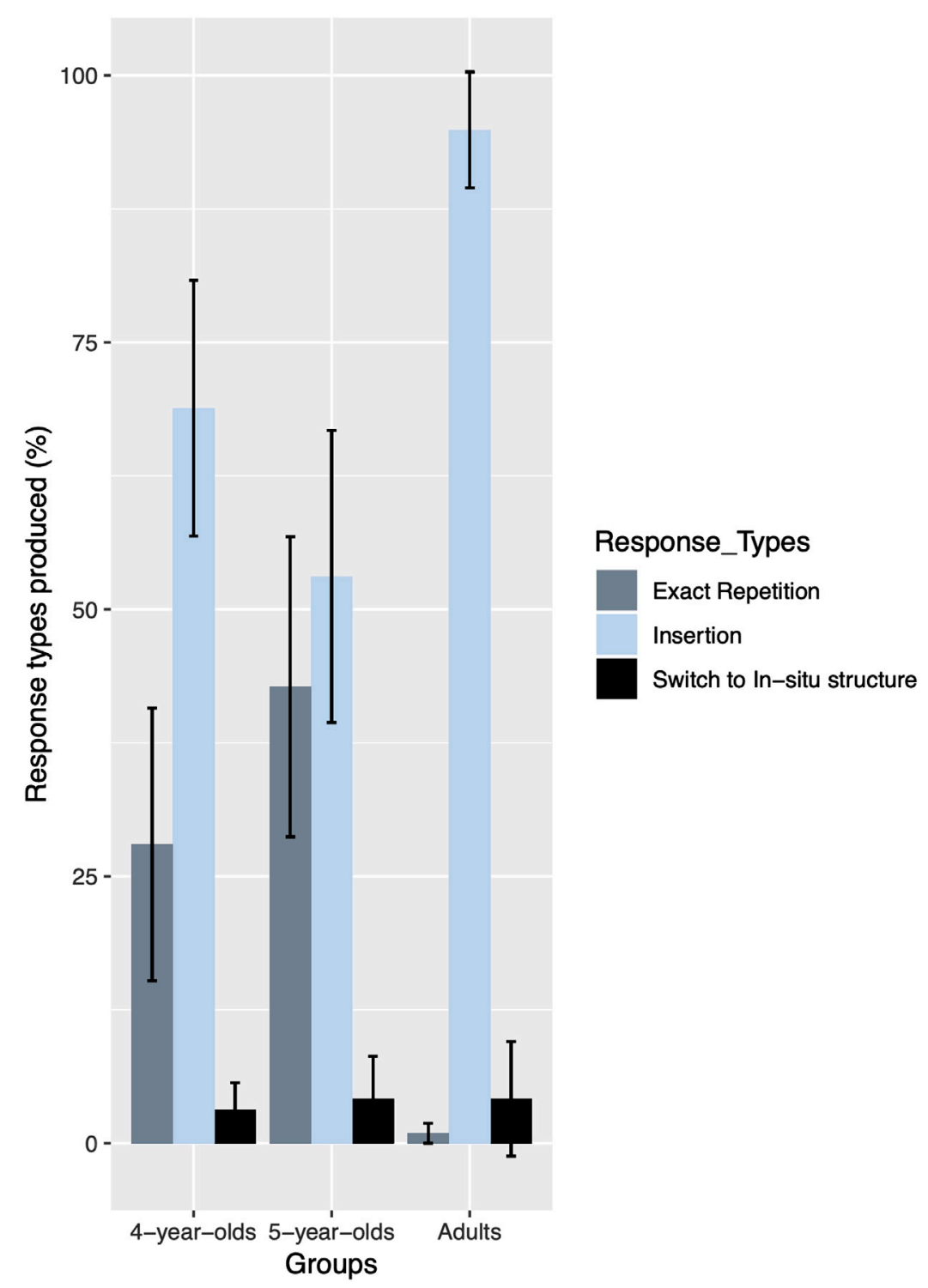

FIGURE 4 | Percentages of response types for the ungrammatical questions produced by the different groups. Error bars indicate a 95\% confidence interval.

keeping the ex-situ structure or by a switch from the ex-situ to the in-situ question without the need to insert additional morphemes. To further explore the non-exact repetitions in the ungrammatical condition we ran a second Generalized Mixed Effects Model with the type of non-exact repetitions as dependent variable $(1=$ insertion $/ 0=$ switch $)$ and Age of participants (4-/5year-olds/Adults) as categorical fixed factors and their interactions. 4-year-olds responses to grammatical questions were assigned to the intercept. The random factors included participants and subjects with random slopes. The summary statistics of the best-fitting model are reported in Table 3. We found a significant effect of Intercept but no significant effect of Age. Also, none of the pairwise comparisons between age groups revealed significant differences $(p>0.05)$, suggesting that participants in all conditions were equally likely to produce more insertions than switches when correcting an ungrammatical question.

We did not find any effect of age in this condition. Participants' corrections of the ungrammatical questions significantly involved the insertion of the clitic morpheme na (and a RP for who-questions) while keeping the ex-situ structure than switching to the in-situ structure for all age groups.

TABLE 3 | Model output of corrections in the ungrammatical ex-situ questions.

\begin{tabular}{lcccc} 
Fixed effect & Estimate & SE & z-value & $\operatorname{Pr}(>|z|)$ \\
\hline Intercept & 3.54794 & 0.40555 & 8.748 & $<0.001^{\star \star \star}$ \\
5-year-olds & -0.31187 & 0.33989 & -0.918 & $<0.5$ \\
Adults & -0.02594 & 0.32224 & -0.081 & $=0.93$
\end{tabular}




\section{DISCUSSION}

In this study we tested children's and adults' performance in an elicited imitation task in which grammatical ex-situ and in-situ questions as well as ungrammatical questions were presented to the participants. All ungrammatical questions contained an initial wh-word but missed the clitic na that obligatorily follows the pronoun in ex-situ questions and the RP that is obligatory in $e x$ situ who-questions. Three main findings were obtained. First, both child groups and the adult group produced a high number of exact repetitions when presented with a grammatical question as compared with when presented with ungrammatical questions. The fact that almost all grammatical questions were exactly repeated by all groups while the proportion of exact repetitions of ungrammatical questions was much lower is strong evidence that our participants are sensitive to the grammaticality vs. ungrammaticality of the questions and thus have the underlying syntactic knowledge. Additionally, the high number of exact repetitions of the grammatical questions does not suggest any difference between ex-situ and in-situ input structures in this task. Second, children produced more exact repetitions of ungrammatical questions than adults without any significant differences between the two child groups. While adults rarely produced exact repetitions of the ungrammatical questions both child groups showed some considerable amount of these ungrammatical reproductions. This may suggest that children at the age that we tested did not apply their grammatical knowledge as robustly as adults did in this task. Third, when correcting the ungrammatical questions all age groups responded with a clearly higher proportion of insertions compared to switches. These findings will be further discussed in the next section.

\section{GENERAL DISCUSSION}

This study has investigated the acquisition of wh-questions in Akan-speaking children. Akan is a language that shows a large degree of optionality between in-situ and ex-situ questions and is therefore an optimal candidate (in addition to previous research on French) to further test the predictions of the Derivational Complexity Hypothesis (DCH) and the Derivational Complexity Metric (DCM) as proposed by Jakubowicz (2004), Jakubowicz (2005). According to this theoretical framework, children are expected to initially prefer the less complex in-situ structure compared to the more complex ex-situ structure if their language allows both options. This study also tests the prediction that the in-situ and ex-situ wh-questions in Akan do not differ in terms of structural complexity (Saah, 1995) and therefore, children learning Akan will not favor one structure over the other on the basis that the in-situ structure is less complex than the ex-situ structure. To this end, we conducted two experiments: an elicited production task and a question repetition task with 4- and 5-year-old Akan-speaking children and adults. Based on the DCM and the DCH, we predicted that children will produce more in-situ than ex-situ questions in the elicited production task, and will more often change an ex-situ question to an in-situ question in the question repetition task.
Based on the alternative linguistic account which argues for equal complexity, we predicted that if the presence of RP in ex-situ whquestions equally makes it less complex as in-situ wh-questions, then children will not show preference for either of the two whquestion structures. Both tasks were also conducted with adults since no previous studies on adult Akan speakers' preference for one structure or the other were available.

First, our results showed that Akan-speaking adults preferred the ex-situ over the in-situ questions in both of our studies: they produced more ex-situ questions in the elicitation task and maintained this structure in their corrections of ungrammatical ex-situ questions in the repetition task. Fouryear old children showed a very similar pattern: they showed the same preference for the ex-situ structure as the adults in the elicitation task and in the repetition task. In contrast to the 4year-olds, the 5-year-olds deviated from the adults' performance in one aspect: they produced more in-situ questions than adults did in the elicitation task while the difference to the 4-year-olds was not significant. However, in their choice of correcting the ungrammatical questions the 5-year-olds were not different from the other age groups as all age groups preferred the correction that maintained the ex-situ structure while inserting the missing grammatical morpheme(s). In general, these findings suggest an overall preference (except for the 5-year-olds in the elicitation task) for the ex-situ over the in-situ question in both of our tasks. This leads to the question why this is the case-especially if one assumes that according to the DCM (Jakubowicz, 2005) this structure is computationally more complex than the in-situ structure. Several points need discussion here.

First, it may be the case that our assumed linguistic analysis is not valid for Akan but that-as proposed by Saah (1995) - no movement is involved in Akan ex-situ questions as indicated by the occurrence of the RP. This is also in line with the prediction that there should be no differences between the in-situ and ex-situ wh-questions with RP, based on the alternative analysis. An indication in our data may support this prediction (i.e., the higher amount of ex-situ in the who-questions that have the overt RP) that we found across all three participant groups. However, the data from Zuckerman and Hulk (2001) from French adults and French-learning 4- and 5-year-olds using a similar elicitation task mirror our findings from Akan which does not support the assumption of crucial structural differences in the formation of ex-situ questions across the two languages.

Second, we cannot exclude task specific effects. The high rates of producing ex-situ questions could be attributed to syntactic priming. Many experiments on sentence production have shown that the choice of a structure by the participants in - for example describing a picture is affected by the structure of a sentence produced before by the experimenter (e.g., Bock, 1986). These syntactic priming effects are already shown by 4 - and 5 -year-old children (Huttenlocher et al., 2004). Most relevant to our study, Hamann (2006) reports priming effects in French children's productions of wh-question in an elicited task with more exsitu questions when the lead in presented by the experimenter contained an ex-situ structure (e.g., demande lui où il habite, ask him where he lives) compared to a neutral lead-in (e.g., demande lui quand; ask him when). Therefore, it is conceivable that 
priming effects have covered any preferences for the in-situ structure in our Study 1 as in all prompts the element that the $w h$-word referred to occurred at the initial position of the embedded clause (see examples 9-11). However, the grammatical sentences in the elicited imitation study of Study 2 also provided no evidence of favoring the in-situ structure which could have led to switches from the ex-situ to the in-situ structure in the grammatical sentences. However, no such tendency occurred in our child data as children repeated the grammatical questions exactly as in the structure that they were presented with.

\section{Implications of These Findings for the Acquisition of Question Formation in Akan}

Our data do not provide evidence that Akan learning children show an overall strong preference for the one or the other question structure that would grossly differ from what we observed in the adult participants of our study. 4-year-olds did not differ significantly from adults in their proportions of in-situ questions and like adults they corrected the ungrammatical questions in most cases by the insertion of the missing morphemes which kept the wh-initial structure of the presented stimulus. Yet, the 4-year-olds also produced a high amount of ex-situ questions (numerically even more than in-situ questions) which clearly indicates that they have the structural knowledge that is necessary to produce both question types. These observed similarities between the 4-year-olds and the adults-also in relation to the responses to the different whpronouns for which no age effects were found - may suggest that 4-year-olds' question production mirrors the frequency relations that they observe in their speech input (if we assume that the adults' question use in our study reflects their daily use of questions and is not purely task induced).

This is in line with previous suggestions that children's acquisition of question formation is item related and does not necessarily generalize to all question types (Hamann, 2006). However, data from spontaneous child-directed speech in Akan would be necessary to obtain a better understanding of the input that children get and of the extent to which the distribution in the input predicts the distribution of the different question types in Akan-learning children's utterances. More research on this topic is definitely necessary.

Surprisingly, the 5-year-old Akan-speaking children were more different from the adults than the 4-year-olds as they produced significantly more in-situ questions than the adults and marginally more in-situ questions than the 4-year-olds in the elicitation study. However, their performance in the elicited imitation task was not different from the 4-year-olds - they showed the same pattern of preferring insertions of grammatical morphemes over structural switches in repairing the ungrammatical questions as the two other age groups.

Looking at the data from Study 1 in more detail suggests that the 5-year-olds not only produced more in-situ questions than the other two groups but that the relation of in-situ vs. ex-situ is numerically reversed in this group, i.e., that they prefer the in-situ over the ex-situ questions. Still, about $40 \%$ of their productions were ex-situ questions in the elicitation task suggesting that like the 4-year-olds, the 5-year-olds have the linguistic competence for both question structures. The question then arises, why the 5-yearolds as a group show slightly different response patterns from the younger children as well as from the adults. Individual differences across the participants do not provide an explanation: Looking at the individual data from Study 1 shows that only 1 adult and 7 (out of 21) 4-year-olds produced numerically more in-situ than ex-situ questions while this was true for 14 (out of 23) 5-year-olds. Accordingly, the overall results from the 5-year-olds reflect a group-wide tendency and are not caused by a few single participants. Instead, we propose the 5-year-olds' performance may reflect an emerging uncertainty concerning potential semantic or pragmatic differences between the two structures. For example, Saah (1995) has proposed that ex-situ and in-situ questions in Akan are different with respect to their information structure. According to this approach ex-situ questions put a stronger focus on the wh-word than in-situ questions, which is also indicated by the obligatory occurrence of the focus marker na directly following the $w h$-word in the ex-situ questions. In line with other research showing that the conditions for pragmatically induced optionality are not easy to acquire for children (Sauermann and Höhle, 2018), we suggest that 5-year-olds may be in the process of figuring out these conditions and therefore may opt for the structurally less complex variant during this process. However, in the question repetition task, no advantage for the insitu questions was observed in this age group-neither in their repetitions of the grammatical questions nor in their corrections of the ungrammatical questions. This difference in the performance of the 5-year-olds across tasks could indicate that the repetition task leaves less room for pragmatic considerations than the elicited production task. However, so far, our interpretation of the results of the 5-year-olds is speculative and more research on adults' use of question structures in Akan-especially on the potential functional differences between the two question types-would be necessary to better understand the children's performance. In any case, our results clearly show that Akan-learning children at the ages tested have both the ex-situ and the in-situ questions in their linguistic repertoires.

This conclusion is also strongly supported by the results from the question repetition task. The huge discrepancy in the number of exact repetitions between the grammatical and the ungrammatical questions found for the children and the adults suggests that 4- to 5-year-old Akan-speaking children are sensitive to the grammatical structure of questions in their language. This finding corroborates findings from other studies that show that sentence repetition tasks are a useful tool to investigate children's grammatical knowledge (e.g., Höhle et al., 2001; Valian and Aubry, 2005; Hamann, 2006). The performance for ungrammatical sentences is specifically interesting in this task since the way in which participants correct the sentences is highly informative about preferences for the choice of constructions when the language allows different options. In the current task participants were instructed to correct questions that they considered to be ungrammatical, but such corrections also occur spontaneously without explicit instruction even in younger children than the groups tested here (Höhle et al., 2001). 


\section{Theoretical Implication(s) of the Current Study for the DCH/DCM}

The Derivational Complexity Metric (DCM) predicts that the insitu wh-questions should be preferred over the ex-situ questions in Akan-speaking children. This prediction is based on the claim that $w h$-questions that involve no internal merge are less complex than those with one or more internal merges. Previous studies on French-speaking children have shown evidence for the role of the DCH/DCM in the acquisition of French. Data from Zuckerman and Hulk (2001) as well as from Hamann (2006) shows that French-speaking 4- to 5-year-old children still use more wh-insitu questions and fewer subject-verb inversion than adults. Younger French-learning children around the age of 2 years have been found to show a clear dominance of in-situ questions over ex-situ questions in their productions, and French children with SLI have shown such a dominance even up to the age of 9 years (Hamann, 2006). Relatedly, Jakubowicz and Strik (2008) found that 4- and 6-year-old French-speaking children preferred to use ex-situ long distance $w h$-questions without verb movement.

Our own data on Akan are only partly compatible with these predictions. The fact that 5-year-olds produced more in-situ whquestions than ex-situ wh-questions lends support to the DCM, which predicts that children will favor wh-questions that involve no internal merge or a lesser number of internal mergeoperations of the wh-element. However, the fact that 5-yearolds but not 4-year-olds produced more in-situ questions than adults challenges the DCM, which predicts an initial emergence of or preference for the (less complex) in-situ structure. This prediction is based on the assumption that the wh-word that occurs at the left periphery of an ex-situ wh-question in Akan undergoes movement (internal merge). However, if one considers the assumption that the presence of a RP in the ex-situ structure makes it equally less complex as the in-situ structure (Demirdache 1991; Shlonsky 1992; Mccloskey, 2002; Alexopoulou, 2006), the DCM would not be applicable and no advantage for the in-situ structure in acquisition would be expected. The data from the 4-year-olds may be interpreted as supporting this alternative linguistic analysis but as mentioned above, similarities in the acquisition data from French and Akan do not support the assumption of different underlying structures in French and Akan ex-situ questions.

If developmental change is considered to reflect a decreasing influence of derivational complexity on children's performance, the differences observed between the 4- and the 5-year-old Akanlearning children are also not compatible with the DCM. However, these differences seem to align with the predictions of the alternative linguistic analysis which did not predict any developmental advantage for one structure over the other since this analysis considers both the in-situ and ex-situ structures to be equal in terms of complexity. In this respect Akan-learning children are not compatible with French-learning children, for whom a decrease in the production of in-situ questions was observed between 4- and 5-years of age (Zuckerman and Hulk, 2001) while our data show an increase of in-situ questions between 4- and 5-year-olds. Which cross-linguistic differences between Akan and French or which methodological details of the tasks used across different studies are relevant for these inconsistent findings are questions for further research. So far, the results from children acquiring Akan seem to diverge from the findings with French children and therefore question the cross-linguistic validity of the DCM as predicting the developmental trajectory in the acquisition of ex- and in-situ questions. One potential explanation for these cross-linguistic differences in development could be that a developmental shift from a preference for the less complex in-situ to the more complex ex-situ question occurs earlier in Akan-learning children than in French-learning children and therefore was not captured in the age range of our sample. As French has more options in question formation than Akan, such a scenario is not implausible. Therefore, research looking at question formation in younger Akan-learning children should also be a task for future research.

\section{CONCLUSION}

The current study presents findings from Akan-speaking children's acquisition of wh-questions based on an experimental elicited production task and an elicited imitation task. Our findings suggest a developmental change in whquestion structure preferences between the ages of 4 and 5 years. We assume that the 4-year-olds' adult-like pattern of performance reflects the frequency of the occurrence of the exsitu and the in-situ structures in their input. The 5-year-olds, however, - unlike adults-show a preference for the in-situ questions. We suggest that this choice is not based on the potential structural differences between the two types of questions but reflects some uncertainty concerning the possible pragmatic functions of the two question types in Akan. That the 5-year-olds choose more often the in-situ option may support the assumption that this is the structurally less complex structure and therefore partially provide evidence for the Derivational Complexity Hypothesis.

\section{DATA AVAILABILITY STATEMENT}

The raw data supporting the conclusions of this article will be made available by the authors, without undue reservation.

\section{ETHICS STATEMENT}

The studies involving human participants were reviewed and approved by Committee on Human Research, Publications and Ethics; School of Medicine and Dentistry, KNUST. Written informed consent to participate in this study was provided by the participants' legal guardian/teachers.

\section{AUTHOR CONTRIBUTIONS}

POO contributed to the design of the work, stimuli construction, data collection, analysis of the data, drafting of the work and 
revising of the manuscript. $\mathrm{BH}$ contributed to the design of the work, interpretation of the data, drafting and revising of the manuscript.

\section{FUNDING}

The work reported on here was part of the MA thesis of the first author, who was funded by a grant from the Erasmus+ Mundus Joint Degree Program in Clinical Linguistics (EMCL+: 20162120/001-001 Project number 574375-EPP-1-2016-1-NLEPPKA1-MDMOB) University of Groningen, University of Potsdam and University of Eastern Finland. The thesis was

\section{REFERENCES}

Akaike, H. (1998). "Information theory and an extension of the maximum likelihood principle," in Selected papers of Hirotugu Akaike. Springer series in statistics (perspectives in statistics). Editors E. Parzen, K. Tanabe, and G. Kitagawa (New York, NY: Springer), 199-213.

Alexopoulou, T. (2006). Resumption in relative clauses. Nat. Lang. Linguist Theory 24 (1), 57-111. doi:10.1007/s11049-005-0898-2

Bates, D. M., Maechler, M., Bolker, B., and Walker, S. (2015). Fitting linear mixed- effects models using lme4. J. Stat. Software 67, 1-48. doi:10.18637/jss. v067.i01

Bayer, J., and Cheng, L. L. (2015). "Wh-in-situ," in The blackwell companion to syntax. Editors M. Everaert and H. van Riemsdijk (Malden, MA: Blackwell Publishing).

Bloom, L., Merkin, S., and Wootten, J. (1982). "Wh"-questions: linguistic factors that contribute to the sequence of acquisition. Child Dev. 53 (4), 1084. doi:10. 1007/978-94-011-6276-0

Bloom, P. (1990). Subjectless sentences in child language. Ling. Inq. 21, 491-504. Available at: http://www.jstor.org/stable/4178692

Boadi, L. A. (1974). Focus marking in Akan. Linguistics 140, 5-57.

Boadi, L. A. (2005). Three major syntactic structures in Akan: interrogatives, complementation and relativisation. Accra, Ghana: Black Mask Ltd.

Bock, J. K. (1986). Syntactic persistence in language production. Cognit. Psychol. 18, 355-387. doi:10.1016/0010-0285(86)90004-6

Bodomo, A., Anderson, J. A., and Dzahene-Quarshie, J. (2009). A kente of many colours: multilingualism as a complex ecology of language shift in Ghana. Socioling. Stud. 3 (3), 357-379. doi:10.1558/sols.v3i3.357

Boll-Avetisyan, N., Omane, P. O., and Kügler, F. (2020). "Speech rhythm in Ghanaian languages: the cases of Akan, Ewe and Ghanaian English" in Proceedings of the international conference on speech prosody, Tokoyo, Japan, May 25-28, 2020, 586-590. Available at: https://doi.org/10.21437/ SpeechProsody.2020-120 (Accessed June 6, 2020).

Brown, R. (1968). The development of wh-questions in child speech. J. Verbal Learn. Verbal Behav. 7, 279-290. doi:10.1016/s0022-5371(68)80002-7

Capdevila i Batet, M. (1993). Children's yes-no and wh-questions. Atlantis $15(1 / 2)$, 35-59. Available at: https://www.jstor.org/stable/41054706

Cheung, C. C. H. (2008). Wh-fronting in Chinese. Doctoral Dissertation. Los Angeles (CA): University of Southern California.

Cheung, C. C.-H. (2014). Wh-fronting and the left periphery in Mandarin. J. East Asian Ling. 23 (4), 393-431. doi:10.1007/s10831-013-9112-4

Chomsky, N. (1995). "Bare phrase structure," in Government and binding theory and the minimalist program. Editor G. Webelhuth (Oxford, United Kingdom: Blackwell).

Chomsky, N. (2001). "Beyond explanatory adequacy," in MIT occasional papers in linguistics 20. (Cambridge, MA: MIT, Department of Linguistics and Philosophy, MITWPL), 1-28.

Citko, B. (2005). On the nature of merge: external merge, internal merge, and parallel merge. Ling. Inq. 36 (4), 475-496. doi:10.1162/002438905774464331

Crain, S., and Thornton, R. (1998). Investigations in universal grammar. Cambridge, MA: MIT Press. jointly supervised by $\mathrm{BH}$ and Gisbert Fanselow, projects $\mathrm{C} 01$ and C03 of the SFB 1287 funded by the Deutsche Forschungsgemeinschaft (DFG, German Research Foundation) Project ID 317633480. We acknowledge the support of the Deutsche Forschungsgemeinschaft and Open Access Publishing Fund of University of Potsdam.

\section{ACKNOWLEDGMENTS}

We thank Alan Langus for his help in the statistical analysis and all participants, headmasters and teachers who supported us in the data collection.

Crisma, P. (1992). On the acquisition of wh in French. Geneva Generative Pap. $155-122$.

De Villiers, J. (1995). Introduction to the special issue on the acquisition of Whquestions. Lang. Acquis. 4, 1-4. doi:10.1207/s15327817la0401\&2_3

Demirdache, H. (1991). Resumptive chains in restrictive relatives, appositives and dislocation structures. Doctoral Dissertation. Cambridge (MA): MIT.

Gazdar, G. (1981). Unbounded dependencies and coordinate structure. Ling. Inq. 12 (2), 155-184. doi:10.1007/978-94-009-3401-6_8

Hamann, C. (2000). "The acquisition of constituent questions and the requirements of interpretation," in The acquisition of syntax. Editors M. A. Friedemann and L. Rizzi (London, United Kingdom: Longman), 170-201.

Hamann, C. (2006). Speculations about early syntax: the production of whquestions by normally developing French children and French children with SLI. Catalan J. Ling. 5, 143-189. doi:10.5565/rev/catjl.82

Hanna, K., and Wilhelm, A. (1992). On the acquisition of WH-questions. Calg. Work. Pap. Ling. 15, 89-98.

Höhle, B., Weissenborn, J., Schmitz, M., and Ischebeck, A. (2001). "Discovering word order regularities: the role of prosodic information for early parameter setting," in Approaches to bootstrapping. phonological, lexical, syntactic and neurophysiological aspects of early language acquisition. Editors J. Weissenborn and B. Höhle (Amsterdam, Netherlands: John Benjamins Publishing Company), Vol. 1, 249-265.

Hulk, A. (1996). The syntax of Wh-questions in child French. Amsterdam Series Child Lang. Dev. 5, 129-172.

Huttenlocher, J., Vasilyeva, M., and Shimpi, P. (2004). Syntactic priming in young children. J. Mem. Lang. 50 (2), 182-195. doi:10.1016/j.jml.2003.09.003

Jakubowicz, C. (2004). "Is movement costly?," in The JEL conference (Journée d'Etudes Linguistiques), University of Nantes, [JEL 2004], May 5-7, Nantes, France.

Jakubowicz, C. (2005). "The language faculty: (Ab) normal development and interface constraints," in Generative approaches to language acquisition, September 8-10, Siena, Italy.

Jakubowicz, C., and Strik, N. (2008). Scope-marking strategies in the acquisition of long distance wh-questions in French and Dutch. Lang. Speech 51, 101-132. doi:10.1177/00238309080510010701

Jakubowicz, C. (2011). Measuring derivational complexity: new evidence from typically developing and SLI learners of L1 French. Lingua 121 (3), 339-351. doi:10.1016/j.lingua.2010.10.006

Klima, E. S., and Bellugi, U. (1966). "Syntactic regularities in the speech of children," in Psycholinguistics papers. Editors J. Lyons and R. J. Wales (Edinburgh, Scotland: Edinburgh University Press) 183-207.

Lartey, N. (2016). Comprehension of object wh-questions in Akan agrammatic speakers: the movement dilemma. (Unpublished Master's thesis). Groningen (Netherlands): University of Groningen.

Lartey, N. (2019). A neurolinguistics approach to pronominal resumption in Akan focus constructions. Doctoral Dissertation. Groningen (Netherlands): University of Groningen.

Lassotta, R., Omaki, A., and Franck, J. (2016). Developmental changes in misinterpretation of garden-path wh-questions in French. Q. J. Exp. Psychol. 69 (5), 829-854. doi:10.1080/17470218.2015.1054845

Lenth, R. (2020). Estimated marginal means, aka least-squares means R package version 1.5.2-1. Available at: https:/CRAN.R-project.org/package=emmeans (Accessed November 4, 2020). 
Marfo, C., and Bodomo, A. (2005). Information structuring in Akan question-word fronting and focus constructions. Stud. Afr. Ling. 34, 179-208.

Mccloskey, J. (2002). "Resumption, successive cyclicity, and the locality of Operations," in Derivation and explanation in the minimalist program. Editors S. D. Epstein and D. T. Seely (Oxford, United Kingdom: Blackwell Publishers), 184-226.

Plunkett, B. (2004). "Early peripheries in the absence of C," in Peripheries syntactic edges and their effect. Editors D. Adger, C. De Cat, and G. Tsoulas (Dordrecht, Netherlands: Kluwer), 383-405.

Pozzan, L., and Valian, V. (2017). Asking questions in child English: evidence for early abstract representations. Lang. Acquis. 24, 209-233. doi:10.1080/10489223.2016. 1187615

Prévost, P., Strik, N., and Tuller, L. (2014). Wh-questions in child L2 French: derivational complexity and its interactions with L1 properties, length of exposure, age of exposure, and the input. Sec. Lang. Res. 30, 225-250. doi:10. $1177 / 0267658313519814$

R Core Team (2018). R: a language and environment for statistical computing. Vienna: Austria: R Foundation for Statistical Computing. Available at: https:// www.R-project.org/ (Accessed July 9, 2020).

Saah, K. (1988). Wh-questions in Akan. J. West Afr. Lang. 18, 17-28.

Saah, K. K. (1995). Studies in Akan syntax, acquisition, and sentence processing. Doctoral Dissertation. Ottawa, ON: University of Ottawa.

Saah, K. K. (2010). "Relative clauses in Akan," in Topics in Kwa syntax. Editors E. O. Aboh and J. Essegbey (London, United Kingdom: Springer), 91-108.

Sauermann, A., and Höhle, B. (2018). Word order in German child language and child-directed speech: a corpus analysis on the ordering of double objects in the German middlefield. Glossa: A J. Gen. ling. 3, 1-32. doi:10.5334/gjgl.281

Shlonsky, U. (1992). Resumptive pronouns as a last resort. Ling. Inq. 23, 443-448.

Smith, M. E. (1933). The influence of age, sex, and situation on the frequency, form and function of questions asked by preschool children. Child Dev. 4 (3), 201-213. doi:10.2307/1125682

Strik, N. (2012). Wh-questions in child bilingual acquisition of French: derivational complexity and cross-linguistic influence. Can. J. Ling. 57 (1), 133-151. doi:10. $1017 /$ s0008413100002231
Stromswold, K. (1995). The acquisition of subject and object Wh-questions. Lang. Acquis. 4, 5-48. doi:10.1080/10489223.1995.9671658

Thornton, R. (1995). Referentiality and wh-movement in child English: juvenile D-linkuency. Lang. Acquis. 4, 139-175. doi:10.1080/10489223. 1995.9671662

Tyack, D., and Ingram, D. (1977). Children's production and comprehension of questions. J. Child Lang. 4 (2), 211-224. doi:10.1017/ s0305000900001616

Valian, V., and Aubry, S. (2005). When opportunity knocks twice: two-year-olds' repetition of sentence subjects. J. Child Lang. 32 (3), 617-641. doi:10.1017/ s0305000905006987

Valian, V., and Casey, L. (2003). Young children's acquisition of wh-questions: the role of structured input. J. Child Lang. 30 (1), 117-143. doi:10.1017/ s0305000902005457

Wimmer, E., Rothweiler, M., and Penke, M. (2017). Acquisition of who-question comprehension in German children with hearing loss. J. Commun. Disord. 67, 35-48. doi:10.1016/j.jcomdis.2017.05.003

Zuckerman, S. (2001). The acquisition of 'optional' movement. Doctoral Dissertation. Groningen (Netherlands): University of Groningen.

Zuckerman, S., and Hulk, A. (2001). Acquiring optionality in French wh-questions: an experimental study. Rev. Québécoise Linguist. 30, 71-97. doi:10.7202/ 000520ar

Conflict of Interest: The authors declare that the research was conducted in the absence of any commercial or financial relationships that could be construed as a potential conflict of interest.

Copyright (C) 2021 Omane and Höhle. This is an open-access article distributed under the terms of the Creative Commons Attribution License (CC BY). The use, distribution or reproduction in other forums is permitted, provided the original author(s) and the copyright owner(s) are credited and that the original publication in this journal is cited, in accordance with accepted academic practice. No use, distribution or reproduction is permitted which does not comply with these terms. 